\title{
Aspherical and Spherical InvA497-Functionalized Nanocarriers for Intracellular Delivery of Anti-Infective Agents
}

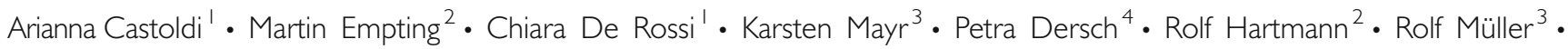 \\ Sarah Gordon ${ }^{1,5}$ (D) Claus-Michael Lehr ${ }^{1,6}$
}

Received: 31 July 2018 / Accepted: 8 October 2018 / Published online: 5 December 2018

(C) The Author(s) 2018

\begin{abstract}
Purpose The objective of this work was to evaluate the potential of polymeric spherical and aspherical invasive nanocarriers, loaded with antibiotic, to access and treat intracellular bacterial infections.

Methods Aspherical nanocarriers were prepared by stretching of spherical precursors, and both aspherical and spherical nanocarriers were surface-functionalized with the invasive protein InvA497. The relative uptake of nanocarriers into HEp-2 epithelial cells was then assessed. Nanocarriers were subsequently loaded with a preparation of the non-permeable antibiotic gentamicin, and tested for their ability to treat HEp-2 cells infected with the enteroinvasive bacterium Shigella flexneri.
\end{abstract}

Guest Editors: Admire Dube

Electronic supplementary material The online version of this article (https://doi.org/| 0. I007/s | | 095-0 | 8-252 I-3) contains supplementary material, which is available to authorized users.

Sarah Gordon

S.C.Gordon@ljmu.ac.uk

Department Drug Delivery, Helmholtz Institute for Pharmaceutical Research Saarland (HIPS), Helmholtz Center for Infection Research (HZI) 66123 Saarbrücken, Germany

2 Department Drug Design and Optimization, HIPS, HZI 66123 Saarbrücken, Germany

3 Department Microbial Natural Products HIPS, HZI 66123 Saarbrücken, Germany

4 Department Molecular Infection Biology, HZI 38124 Braunschweig, Germany

5 School of Pharmacy and Biomolecular Sciences, Liverpool John Moores University Liverpool L3 3AF, UK

6 Department of Pharmacy, Saarland University 66123 Saarbrücken, Germany
Results InvA497-functionalized nanocarriers of both spherical and aspherical shape showed a significantly improved rate and extent of uptake into HEp-2 cells in comparison to nonfunctionalized nanocarriers. Functionalized and antibioticloaded nanocarriers demonstrated a dose dependent killing of intracellular S. flexneri. A slight but significant enhancement of intracellular bacterial killing was also observed with aspherical as compared to spherical functionalized nanocarriers at the highest tested concentration.

Conclusions InvA497-functionalized, polymer-based nanocarriers were able to efficiently deliver a non-permeable antibiotic across host cell membranes to affect killing of intracellular bacteria. Functionalized nanocarriers with an aspherical shape showed an interesting future potential for intracellular infection therapy.

KEY WORDS aspherical nanoparticles · AOT-gentamicin . bacteriomimetic nanocarriers · intracellular infection · invasin

\section{ABBREVIATIONS}

\begin{tabular}{|c|c|}
\hline AOT-gentamicin & $\begin{array}{l}\text { Gentamicin bis(2-ethylhexyl) } \\
\text { sulfosuccinate sodium salt }\end{array}$ \\
\hline$A R$ & Aspect ratio \\
\hline Asph & Aspherical nanocarriers \\
\hline AsphG & $\begin{array}{l}\text { Aspherical AOT-gentamicin-loaded } \\
\text { nanocarriers }\end{array}$ \\
\hline Asphl & $\begin{array}{l}\text { Aspherical InvA497-functionalized } \\
\text { nanocarriers }\end{array}$ \\
\hline AsphlG & $\begin{array}{l}\text { Aspherical AOT-gentamicin-loaded, } \\
\text { InvA497-functionalized nanocarriers }\end{array}$ \\
\hline $\mathrm{BCA}$ & Bicinchoninic acid \\
\hline CFU & Colony forming units \\
\hline DAPI & 4',6-diamidino-2-phenylindole \\
\hline DLS & Dynamic light scattering \\
\hline DMTMM & $\begin{array}{l}\text { 4-(4,6-Dimethoxy-1,3,5-triazin-2-yl)-4- } \\
\text { methylmorpholinium chloride }\end{array}$ \\
\hline
\end{tabular}




$\begin{array}{ll}\text { EE\% } & \text { encapsulation efficiency } \\ \text { FACS } & \text { Fluorescence-activated cell sorting } \\ \text { FA-PLGA } & \text { Fluoresceinamine-PLGA } \\ \text { FCS } & \text { Fetal calf serum } \\ \text { FSC } & \text { Forward scatter } \\ \text { InvA497 } & 497 \text { amino acid length fragment of the } \\ & \text { C-terminal region of invasin } \\ \text { LC\% } & \text { Loading capacity } \\ \text { MOI } & \text { Multiplicity of infection } \\ \text { NTA } & \text { Nanoparticle tracking analysis } \\ \text { PLGA } & \text { Poly (lactic-co-glycolic acid) } \\ \text { PVA } & \text { Polyvinyl alcohol } \\ \text { RPMI } & \text { Roswell Park Memorial Institute } \\ \text { SE } & \text { Standard error of the mean } \\ \text { SEM } & \text { Scanning electron microscopy } \\ \text { Sph } & \text { Spherical nanocarriers } \\ \text { SphG } & \text { Spherical AOT-gentamicin-loaded } \\ & \text { nanocarriers } \\ \text { Sphl } & \text { Spherical InvA497-functionalized } \\ & \text { nanocarriers } \\ \text { SphlG } & \text { Spherical AOT-gentamicin-loaded, } \\ \text { SSC } & \text { InvA497-functionalized nanocarriers } \\ & \text { Side scatter }\end{array}$

\section{INTRODUCTION}

While delivery of anti-infective drugs using nanocarriers is an attractive option for the treatment of infections, several factors may act to limit the efficacy of this strategy. The specific location of bacteria constitutes one such obstacle - many common enteropathogenic bacteria such as Salmonella, Shigella and Yersinia spp. are able to invade and replicate inside host cells, where, due to the common poor membrane permeability of many anti-infective agents, they prove difficult to reach $(1,2)$. Incorporation of drug candidates into particulate nanocarriers functionalized with invasive moieties to enhance cellular uptake is a potential way to overcome this delivery problem. In this respect, the use of bacterial proteins which naturally mediate the invasion of bacteria into mammalian cells has been reported as a promising means of enhancing the permeation of carrier systems, and potentially increasing the intracellular efficacy of their drug loads (3-6).

A particularly interesting candidate in this category is invasin, a well-characterized outer membrane invasion protein expressed on the surface of Tersinia pseudotuberculosis and Yersinia enterocolitica, which mediates an efficient entry of the bacteria into eukaryotic cells through interaction with $\beta_{1}$ integrin receptors $(7,8)$. The last 497 amino acids of the $C$-terminal region of invasin have been found to be particularly important for receptor binding and intracellular uptake (8). Dersch et al. were able to produce and purify such a $C$-terminal, cell-invasive fragment of invasin, referred to as
InvA497 (8); gentamicin-loaded liposomes surface functionalized with InvA497 were further demonstrated to be able to reach and kill intracellular bacteria located in various epithelial sub-cellular compartments $(3,9)$.

The potential of invasin and the invasin fragment InvA497 is therefore clear, however the full capacity of so-called bacteriomimetic systems, functionalized with such cell invasion-promoting molecules, still remains to be fully explored. In the first instance, while surface functionalization of comparatively more robust, polymer-based carrier systems with invasin has been shown to improve their cellular uptake $(5,6)$, drug loading of such systems and investigation of their ability to affect intracellular delivery of actives has not been investigated in-depth. In addition to probing the ability of invasin to mediate effective intracellular delivery of drug loads from carrier systems composed of a variety of materials, there is also a need to investigate the role of other factors, such as the shape of these carrier systems, on delivery efficacy.

There is clear evidence of the importance of the shape of colloidal structures in biological interactions, including the shape variation of bacteria themselves $(10,11)$; however, due to the fact that most nanoparticles employed for drug delivery are inherently spherical (as a result of the nature of utilized preparation procedures), the role of their shape in mediating effective drug delivery has largely been unexplored to date $(12,13)$. Studies have shown the possibility of using particles with an aspherical shape to alter circulation time and biodistribution $(14,15)$ as well as cellular internalization and trafficking (16-20), subsequently influencing the interaction of the particle with its target. With respect to cell uptake, particle shape effects have been mainly studied in phagocytic cells such as macrophages, as well as non-phagocytic epithelial cells and in most cases, the uptake of elongated particles was significantly inhibited in comparison to spherical controls $(19,21,22)$. In contrast, initial studies looking at the influence of shape on uptake by non-phagocytic epithelial cells have shown that aspherical nanoparticles surface-functionalized with biotin had an enhanced uptake into human enterocytes (23), and that trastuzumab-coated nanorods had a higher uptake in breast cancer cell lines than spherical or disk-shaped nanoparticles (24). The efficacy of loaded aspherical systems has also been investigated, with varying results. Kolhar et al. have shown a positive effect of elongated particles with surface-adsorbed antibody or protein on targeting the endothelium, for delivery of chemotherapeutics (25). Hinde et al. have also shown the advantages of nanoparticles with high aspect ratios, such as 'worms' and rods, for delivery of doxorubicin into the nuclei of epithelial breast cancer cells, demonstrating the impact of various nanocarrier shapes on anticancer formulations (26). When investigated for their potential as vaccine delivery systems however, elongated nanoparticles were shown to be inferior to spherical nanoparticles with respect to dendritic cell activation (18). Therefore, although 
shape has a clear impact on delivery system behavior, the nature of this impact may vary. As such, the effect of aspherical shaped delivery systems must be carefully evaluated in relation to factors including the surface composition of the particulate system, the specific target cell, the drug delivered and the nature of the specific delivery system application. As mentioned above, the uptake of aspherical systems has been investigated in a variety of cell types, however to date the efficacy of drug-loaded aspherical nanoparticles has mainly been studied for delivery of chemotherapeutics; intracellular delivery of other drug classes, such as antibiotics, using aspherical nanoparticles has not yet been investigated to the best of the authors' knowledge.

Therefore, the objectives of this study were to investigate the cellular uptake and efficacy of drug-loaded, polymeric bacteriomimetic systems against intracellular bacteria, and to determine the influence of shape on the physico-chemical characteristics of these systems. For this purpose, InvA497functionalized polymeric nanoparticles with spherical and aspherical morphologies were prepared. Bacteriomimetic systems were shown to have a greater uptake into cells of the HEp-2 human epithelial cell line than non-functionalized nanoparticles, regardless of shape. HEp-2 epithelial cells, which could be reproducibly infected with intracellular Shigella flexneri, were then used as a model for efficacy testing of bacteriomimetic systems loaded with a lipophilic preparation of the antibiotic gentamicin. InvA497-functionalized and drug-loaded systems were able to penetrate into infected cells and kill intracellular S. flexneri, while negligible cell uptake and bacterial killing was demonstrated by the non-functionalized nanoparticles. Moreover, a slight but significant improvement in bacterial killing was found following treatment with high dose aspherical bacteriomimetic systems, in comparison to spherical. The current work therefore represents the first study into the impact of modifying the surface of drug-loaded, polymeric nanoparticles through the use of the bacteria-derived invasion molecule InvA497, in combination with investigation of carrier system shape effects.

\section{MATERIALS AND METHODS}

\section{Materials}

For the preparation and storage of spherical and aspherical nanoparticles, poly(lactic-co-glycolic-acid) (PLGA, Resomer RG 503 H, lactic/glycolic acid 50/50 wt/wt; molecular weight $40.3 \mathrm{kDa}$; inherent viscosity $0.41 \mathrm{dl} / \mathrm{g}$; Evonik Industries AG, Darmstadt, Germany), polyvinyl alcohol (PVA; Mowiol® 4-88, Kuraray Specialties Europe GmbH, Frankfurt, Germany), trehalose (Sigma-Aldrich, Steinheim, Germany) and glycerol (Sigma-Aldrich, Steinheim, Germany) were used. As a coupling agent, 4-(4,6-
Dimethoxy-1,3,5-triazin-2-yl)-4-methylmorpholinium chloride (DMTMM, Sigma Aldrich, Steinheim, Germany) was employed. For in vitro cell experiments HEp-2 cells (ATCG, Manassas, USA), Roswell Park Memorial Institute (RPMI) 1640 medium (Gibco, Carlsbad, USA), fetal calf serum (FCS, Lonza, Cologne, Germany), rhodamine-labeled Ricinus communis Agglutinin I (Vector Laboratories, Inc., Burlingame, CA, USA), paraformaldehyde (Electron Microscopy Sciences, Hatfield, USA) and 4',6-diamidino-2phenylindole (DAPI, stock $1 \mathrm{mg} / \mathrm{ml}$; LifeTechnologies ${ }^{\mathrm{TM}}$, Darmstadt, Germany) were purchased. For quantification of gentamicin, o-phthaldialdehyde reagent (OPA, Sigma Aldrich, Steinheim, Germany) was used. As an intracellular bacterium for infection studies, S. flexneri (clinical strain M90 T) was kindly supplied by the Department of Molecular Infection Biology, HZI, Braunschweig, Germany. For efficacy studies gentamicin (Sigma-Aldrich, Steinheim, Germany) and Triton X-100 (Sigma Aldrich, Steinheim, Germany) were purchased. Distilled de-ionized water with conductivity of less than $18.2 \mathrm{M} \Omega / \mathrm{cm}$ at $25^{\circ} \mathrm{C}$ and organic solvents of HPLC grade were used for all experiments.

\section{Preparation of Spherical Nanoparticles}

Spherical PLGA nanoparticles were prepared using a single emulsion method (27), employing a mixture of PLGA and fluoresceinamine-PLGA (FA-PLGA) prepared according to Weiss et al. (28) at a 0.25:0.75 weight ratio. Briefly, the polymer mixture was dissolved in $2 \mathrm{ml}$ ethyl acetate $(20 \mathrm{mg} / \mathrm{ml})$ and sonicated with $4 \mathrm{ml}$ of a $2 \%(w / v)$ PVA solution at $12 \mathrm{~W}$ for $30 \mathrm{~s}$ (Digital sonifier 450, Branson Ultrasonic Corporation, Danbury, USA). After adding $15 \mathrm{ml}$ of water, the formed emulsion was left to stir overnight to allow for solvent evaporation. Excess PVA was then removed from the nanoparticle dispersion by centrifugation $\left(10,000 \mathrm{~g}\right.$ for $12 \mathrm{~min}$ at $12^{\circ} \mathrm{C}$ ). Nanoparticle suspensions were then stored at $4^{\circ} \mathrm{C}$ for maximum 1 week prior to further use, or freeze dried (Alpha 2-4 LSC, Christ, Osterode am Harz, Germany) with $0.31 \mathrm{mg} / \mathrm{ml}$ of trehalose as cryoprotectant and stored at room temperature.

\section{Aspherical Nanoparticle Preparation}

Aspherical nanoparticles were prepared according to a previously described film stretching method $(29,30)$. Briefly, $0.1 \%$ $(w / v)$ of spherical nanoparticles was mixed with a solution of $10 \%$ PVA and $2 \%(v / v)$ glycerol. The resulting dispersion was dried overnight in a mold in order to create a flat, dry film. Sections of the film were then immobilized in an in-house fabricated stretching machine, which was immersed in mineral oil at $54^{\circ} \mathrm{C}$ (above the PLGA glass transition temperature). The films were then stretched longitudinally to twice their original length. The stretched films were allowed to cool 
down, and after removing the excess oil by washing with isopropanol, were dissolved in water. PVA was removed and the stretched, aspherical nanoparticles purified using multiple cycles of high speed centrifugation $(16,000 \mathrm{~g}$ for $20 \mathrm{~min}$ at $12^{\circ} \mathrm{C}$ ) followed by centrifugation (5-6 cycles of $10 \mathrm{~min}$ at $1179 \mathrm{~g}, 4^{\circ} \mathrm{C}$ ) with Centrisart ${ }^{\circledR}$ ultrafiltration tubes (300,000 molecular weight cut off, Sartorius, Göttingen, Germany). Aspherical nanoparticles were stored at $4^{\circ} \mathrm{C}$ until further use.

\section{Characterization of Nanoparticles}

The morphology of spherical and aspherical nanoparticles was characterized using scanning electron microscopy (SEM, Zeiss EVO HD 15, Carl Zeiss AG, Oberkochen, Germany) employing an accelerating voltage of $5 \mathrm{kV}$. Prior to analysis samples were diluted and dried overnight, before being sputter coated (Quorum Q150R ES, Quorum Technologies Ltd., Laughton, United Kingdom) with gold. Physical characterization of spherical nanoparticle dispersions was performed by dynamic light scattering (DLS) using a Zetasizer Nano (Malvern Instruments Ltd., Worcestershire, United Kingdom). For aspherical nanoparticles, the common shape descriptors of major and minor axis length and aspect ratio (AR) were measured from SEM images using ImageJ software (Fiji).

\section{Surface Functionalization}

Nanoparticles were functionalized with InvA497, a fragment of the $\mathcal{Y}$. pseudotuberculosis invasin protein, consisting of 497 amino acids of the parent protein $C$-terminus (3,31).

The InvA497 fragment was purified as described previously (31). In the case of spherical nanoparticles, a volume of $1 \mathrm{ml}$ of spherical PLGA nanoparticles was diluted with $0.9 \mathrm{ml}$ water, and incubated for $2 \mathrm{~h}$ with a $5 \mathrm{mg} / \mathrm{ml}$ solution of the carboxyl group activating agent DMTMM at room temperature. Afterwards the dispersion was again diluted in water and InvA497 was added to a final concentration of $320 \mu \mathrm{g} / \mathrm{ml}$, followed by overnight stirring in an ice bath. To remove excess DMTMM and unbound InvA497, nanoparticles were then centrifuged three times in Centrisart ${ }^{\circledR}$ tubes as mentioned above, at $1605 \mathrm{~g}$ and $4^{\circ} \mathrm{C}$, for $10 \mathrm{~min}$ each cycle.

In order to produce InvA497-functionalized aspherical nanoparticles, spherical PLGA nanoparticles were first incubated for $2 \mathrm{~h}$ with DMTMM as described above. Particles were then immobilized in PVA-glycerol films, and stretched using the stretching apparatus as described above. A $2.5 \mathrm{ml}$ volume of the resulting aspherical nanoparticle dispersion was then stirred overnight in an ice bath with InvA497 (concentration $320 \mu \mathrm{g} / \mathrm{ml}$ ). To remove unbound InvA497, the dispersion was then centrifuged in Centrisart ${ }^{\circledR}$ tubes at $1605 \mathrm{~g}$ and $4^{\circ} \mathrm{C}$, for three cycles of $10 \mathrm{~min}$ each.

\section{Quantification of Functionalized InvA497}

The amount of InvA497 coupled to spherical and aspherical nanoparticles was quantified using a bicinchoninic acid (BCA) kit (QuantiPro ${ }^{\text {TM}}$; Sigma-Aldrich, Steinheim, Germany), in accordance with the manufacturer's instructions and as previously described $(3,32)$.

\section{Nanoparticle Counting}

The number of spherical and aspherical InvA497functionalized nanoparticles within defined samples was counted using nanoparticle tracking analysis (NTA, NanoSight LM 10, Malvern Instruments Ltd., Worcestershire, United Kingdom). After appropriate dilution, the concentration of nanoparticles within a sample was first calculated by the NTA software, and then converted into a number of nanoparticles in the total dispersion. The extrapolated number of nanoparticles was then combined with the quantified amount of InvA497 present on nanoparticle surfaces, and used to estimate the number of InvA497 molecules per nanoparticle as well as the protein density on nanoparticle surfaces.

\section{Generation of 3D Model}

A 3D model of InvA497-decorated spherical and aspherical nanoparticles was generated via a self-written script for PovRay 3.7. To this end, X-ray coordinates of InvA497 (PDB ID: 1CWV) (33) were exported and scaled to the PovRay format using YASARA structure (YASARA Biosciences) (34). After modeling of the nanoparticulate shapes, the calculated numbers of 198 and 235 InvA497 molecules were randomly distributed on the aspherical and spherical objects, respectively. The picture was rendered with subsurface light scattering turned on.

\section{Cell Culture}

Cells of the human larynx carcinoma-derived HEp-2 cell line were employed for both uptake and efficacy studies. HEp-2 cells (passage number 10-18) were cultured in $75 \mathrm{~cm}^{2}$ flasks using RPMI 1640 medium, supplemented with 10\% FCS. Cells were incubated at $37^{\circ} \mathrm{C}$ and $5 \% \mathrm{CO}_{2}$ and medium was changed every two to three days. Cells were split upon $80 \%$ confluency.

\section{Uptake Studies}

HEp-2 cells were seeded onto 24-well cell culture plates the day before conduction of uptake studies. For the studies themselves, cells were incubated with non-loaded spherical and aspherical nanoparticles, with or without surface coupled InvA497 $(455 \mu \mathrm{g} / \mathrm{ml}$ of PLGA and, where appropriate, 
$60 \mu \mathrm{g} / \mathrm{ml}$ of InvA497 per sample) for time points ranging between 1 and $5 \mathrm{~h}$, at $37^{\circ} \mathrm{C}$ and $5 \% \mathrm{CO}_{2}$.

For confocal imaging of nanoparticle-treated cell samples, the supernatant was first removed from culture plate wells and cells were washed twice in order to remove non-internalized nanoparticles. Cell membranes were then stained with $20 \mu \mathrm{g} / \mathrm{ml}$ rhodamine-labeled Ricinus communis Agglutinin I. After fixation with 3\% paraformaldehyde in phosphate buffered saline (PBS), cell nuclei were stained with DAPI (stock $1 \mathrm{mg} / \mathrm{ml}$ ) diluted 1:50000 in PBS. Cells were imaged using confocal laser scanning microscopy (CLSM, Leica TCS SP 8; Leica, Mannheim, Germany). Analysis of gained images was performed using LAS X software (Leica Application Suite X; Leica, Mannheim, Germany).

For fluorescence-activated cell sorting (FACS) analysis, after removing cell supernatants and washing to remove extracellular nanoparticles, cells were detached from 24-well culture plates by incubating with $100 \mu \mathrm{l}$ of $0.05 \%$ trypsin-EDTA (1x, Gibco, Carlsbad, USA) for $10 \mathrm{~min}$ at $37^{\circ} \mathrm{C}$. A $900 \mu \mathrm{l}$ volume of $2 \%$ FCS in PBS was then added, and cell samples were centrifuged at $257 \mathrm{~g}$ for $5 \mathrm{~min}$ at $4^{\circ} \mathrm{C}$. Cell pellets were then resuspended in a $600 \mu \mathrm{l}$ volume of $2 \%$ FCS in PBS, and stored at $4^{\circ} \mathrm{C}$ until FACS analysis. Flow cytometry was performed using a BD LSRFortessaTM (BD Biosciences, Heidelberg, Germany) using BD FACSDiva ${ }^{\text {TM }}$ Software v8.0.1. Forward scatter (FSG) and side scatter (SSG) were collected for live cells within samples, using an untreated negative control for reference. From living cell populations, green fluorescence data (ex: 488 nm, filter: 530/30) were collected on a minimum of 10,000 events (cells) per sample.

To determine the energy dependence of nanoparticle uptake, the same uptake study procedure and FACS analysis was applied, after also incubating the nanoparticles with HEp-2 cells at $4^{\circ} \mathrm{C}$.

\section{Preparation of AOT-Gentamicin Loaded Nanoparticles}

Spherical and aspherical nanoparticles were loaded with a lipophilized preparation of gentamicin (gentamicin bis(2ethylhexyl) sulfosuccinate sodium salt, or AOT-gentamicin), prepared according to Imbuluzqueta et al. $(35,36)$. Spherical AOT-gentamicin nanoparticles (SphG) were first prepared as described above, incorporating $3 \mathrm{mg}$ of the ionic AOTgentamicin preparation into the PLGA solution prior to emulsion formation. The unentrapped AOT-gentamicin was removed using centrifugation and precipitation $(10,000 \mathrm{~g}$ for 12 min at $12^{\circ} \mathrm{G}$ ). Aspherical AOT-gentamicin (AsphG) nanoparticles were prepared by stretching of SphG nanoparticles, as described above. Where required, InvA497 was then coupled on the surface in order to produce functionalized spherical and aspherical nanoparticles loaded with AOT-gentamicin (respectively SphIG and AsphIG), also as described above.
The amount of AOT-gentamicin encapsulated in nanoparticles was quantified as described by Imbuluzqueta et al. (36) using a fluorometric method based on the use of OPA. The amount of encapsulated AOT-gentamicin within samples together with the initial amount of drug used during nanoparticle preparation was used to calculate nanoparticle encapsulation efficiency $(\mathrm{EE} \%)$. After determining the dry mass of nanoparticle samples, the loading capacity $(\mathrm{LC} \%$ ) was calculated as the amount of encapsulated drug related to the total sample weight.

\section{In Vitro Drug Release Testing}

Multiple samples of nanoparticle formulations containing $69.36 \mu \mathrm{g} / \mathrm{ml}$ of AOT-gentamicin were centrifuged and resuspended in $7 \mathrm{ml}$ of PBS pH 7.4 in order to achieve sink conditions. Formulation samples were incubated at $37^{\circ} \mathrm{C}$ under stirring for $48 \mathrm{~h}$; at various time points, 2 samples of each nanoparticle formulation were taken and centrifuged $(1680 \mathrm{~g}, 10 \mathrm{~min})$ in order to sediment the drug-containing nanoparticles. From the produced supernatant, the amount of released AOT-gentamicin was measured using the aforementioned fluorometric method (36). Release testing was conducted in triplicate, independent experiments.

\section{Efficacy Studies}

The ability of drug-loaded nanoparticle systems to kill intracellular bacteria was tested in S. flexneri-infected HEp-2 epithelial cells. After seeding and culturing HEp-2 cells in a 24-well culture plate for $24 \mathrm{~h}$, the cells were infected with $S$. flexneri dispersed in RPMI 1640 medium as described in the Supplementary Material (MOI of 25:1, CFU approximately $\left.1.8 \times 10^{3}\right)$. Cells were then washed with PBS and incubated for $2 \mathrm{~h}$ with RPMI medium containing $50 \mu \mathrm{g} / \mathrm{ml}$ of gentamicin for extracellular bacteria killing. After killing of extracellular bacteria, infected HEp-2 cells were incubated with the InvA497-functionalized or non-functionalized, spherical and aspherical AOT-gentamicin loaded nanoparticles $(120 \mu \mathrm{g} / \mathrm{ml}$ of AOT-gentamicin, $49 \mu \mathrm{g} / \mathrm{ml}$ of InvA497 and $3.7 \mathrm{mg} / \mathrm{ml}$ of PLGA/FA-PLGA where appropriate - standardized by employing drug-free functionalized or drug-free non-functionalized nanoparticles of corresponding shape where necessary) or with AOT-gentamicin alone, for $3 \mathrm{~h}$ at $37^{\circ} \mathrm{C}$ and $5 \%$ $\mathrm{CO}_{2}$. HEp-2 cells were then lysed using $0.01 \%$ Triton X-100 and the cell lysate was plated in sterile agar plates in serial dilutions. Plated lysates were incubated overnight at $37^{\circ} \mathrm{C}$; after counting $S$. flexneri bacterial colonies and multiplication by relevant dilution factors, the final number of colony forming units (CFU) calculated from each cell lysate was expressed as the percentage of remaining intracellular bacteria (relative to the CFU of bacteria used for initial infection the inoculum). Values for each formulation treatment group 
were then normalized to the percentage of remaining intracellular bacteria in untreated cell samples, and expressed as a percentage of bacterial killing.

\section{Statistical Analysis}

Where appropriate, data are expressed as mean \pm standard error of mean (SE). Also where appropriate, data was analyzed using SigmaPlot Version 11 (Systat Software Inc., San Jose, CA, USA) for statistical significance. Comparisons between groups were performed using Student's t test (twosided) or one-way ANOVA with post-hoc Bonferroni adjustment for experiments with more than two subgroups. Results were considered statistically significant at $p$ values $<0.05$.

\section{RESULTS}

\section{Nanoparticle Preparation and Characterization}

Spherical (Sph) nanoparticles produced using a mixture of PLGA and FA-PLGA were stretched, employing a film stretching method established by Champion et al. (29), in order to produce aspherical (Asph) nanoparticles. A change in the shape of stretched nanoparticles was confirmed by SEM imaging (Fig. 1a and b). Nanoparticle dimensions were then determined by SEM image analysis for Asph nanoparticles, giving values of approximately $300 \mathrm{~nm}$ and $110 \mathrm{~nm}$ for major and minor particle axes respectively (Fig. 1c), and an AR of 2.6. Sph nanoparticles were by comparison approximately $164.8 \mathrm{~nm}$ in diameter, as determined by DLS (Fig. 1c).

Functionalization of both aspherical and spherical nanoparticles with InvA497, a $C$-terminal fragment of the bacterial invasion protein invasin, was then achieved by reacting amine functionalities of InvA497 with free PLGA carboxyl groups on nanoparticle surfaces (Fig. 2a). A continued aspherical and spherical particle shape following the coupling procedure was confirmed using SEM imaging, and the size of both functionalized particle formulations was found to be comparable to non-functionalized nanoparticles (Fig. S1). Coupling conditions were optimized so that both the total amount and the number of coupled InvA497 molecules/nanoparticle were comparable for aspherical and spherical nanoparticles (formulations AsphI and SphI respectively, Fig. 2b). Approximately 200 molecules of InvA497 were determined to be present on the surface of each AsphI or SphI nanoparticle. Using published X-ray coordinates of InvA497 (PDB ID: 1CWV) (33) as well as the experimentally determined scales and proportions of both particle types (vide supra), illustrative $3 \mathrm{D}$ models of the corresponding surface-modified carrier systems were generated (Fig. 2c).

Assuming that particle surface areas can be calculated by applying the generic formulas of the corresponding geometric
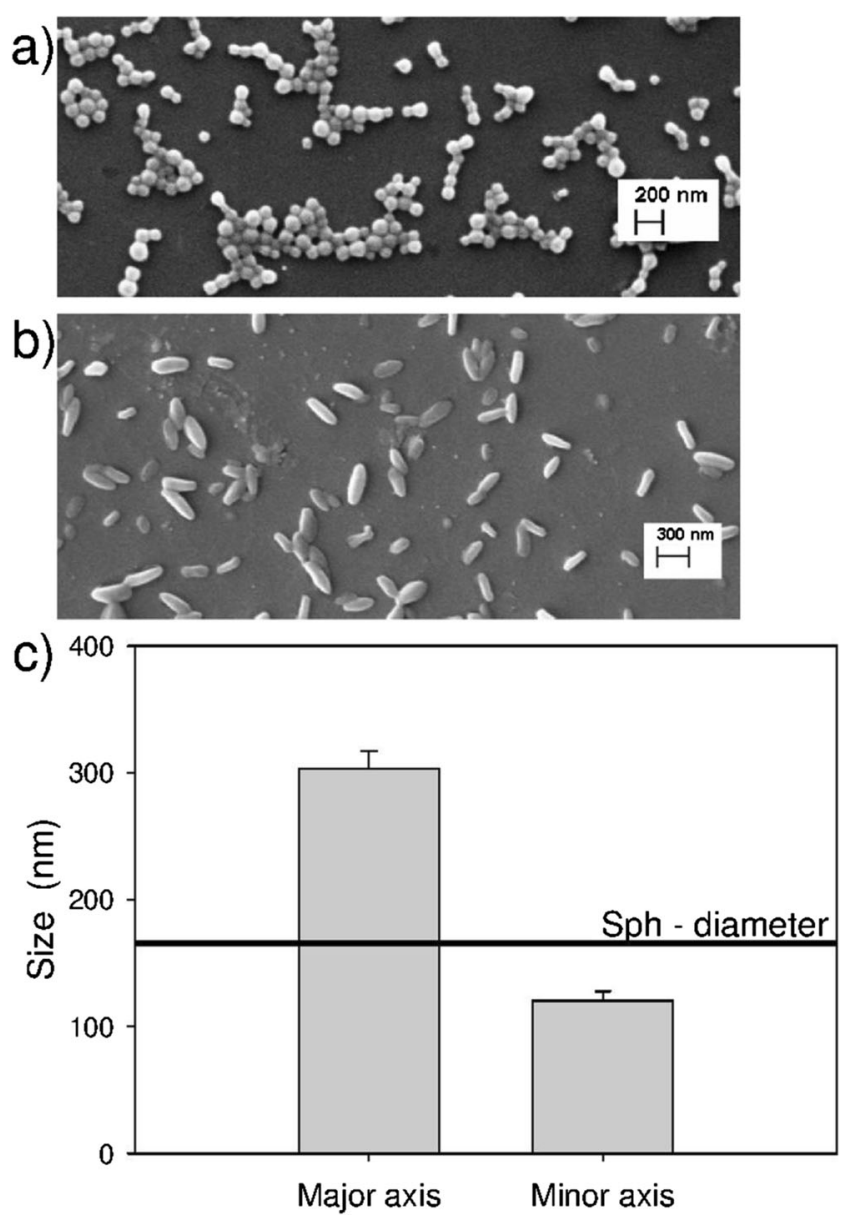

Fig. I Characterization of spherical and aspherical nanoparticles. SEM images of spherical (a) and aspherical (b) nanoparticles; (c) size of the aspherical nanoparticles ('Asph') with respect to their major and minor axis, with the mean diameter of spherical nanoparticles ('Sph' - $164.8 \pm 8.3 \mathrm{~nm}$ ) given as reference. Results represent the mean \pm SE $(n=200)$.

shapes (Sph: sphere and Asph: spheroid) and that each InvA497 molecule occupies a circular area dictated by its gyration radius, the degree of particle surface coverage was estimated. According to these assumptions, approximately $39 \%$ of the surface of optimized AsphI and SphI nanoparticles is occupied by the InvA497 protein (for calculation see Supplementary Material).

\section{Nanoparticle Uptake}

Prior to carrying out uptake studies, the cytotoxicity of functionalized and non-functionalized aspherical and spherical nanoparticles was preliminarily tested in a HEp-2 epithelial cell model (Fig. S2). Functionalized and non-functionalized aspherical and spherical nanoparticles were incubated with HEp-2 cells and the uptake was assessed by both CLSM (Fig. 3) and FACS analysis (Fig. 4).

Confocal images clearly demonstrated an increased uptake of AsphI and SphI (Fig. 3a and b respectively) in comparison 


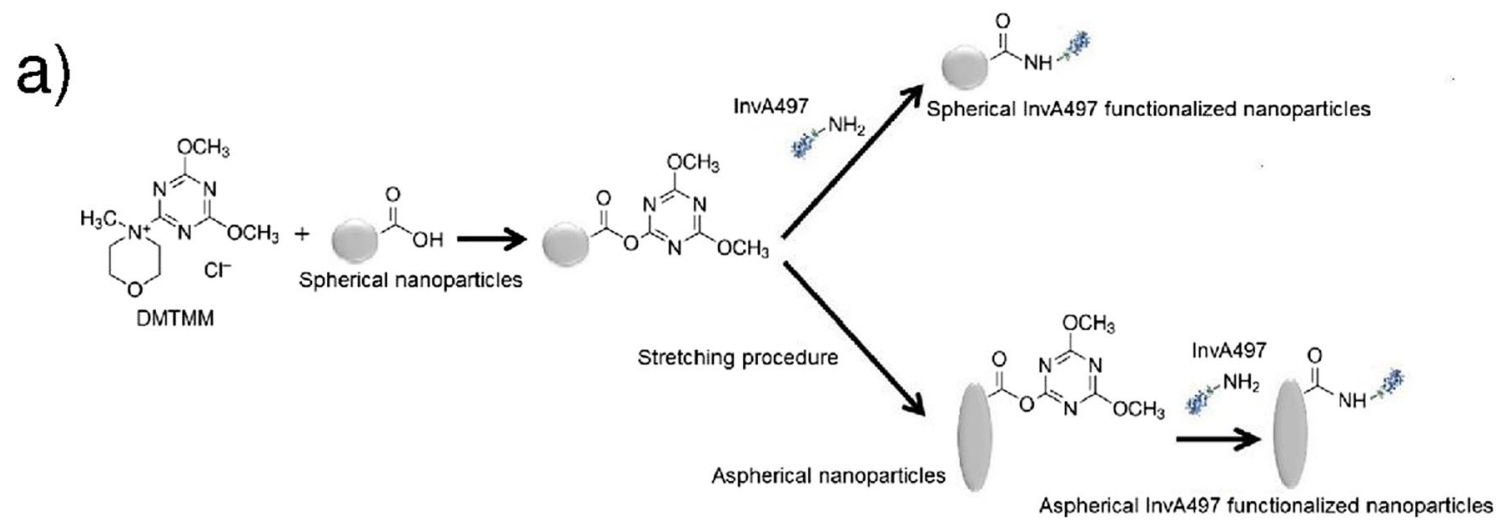

b)
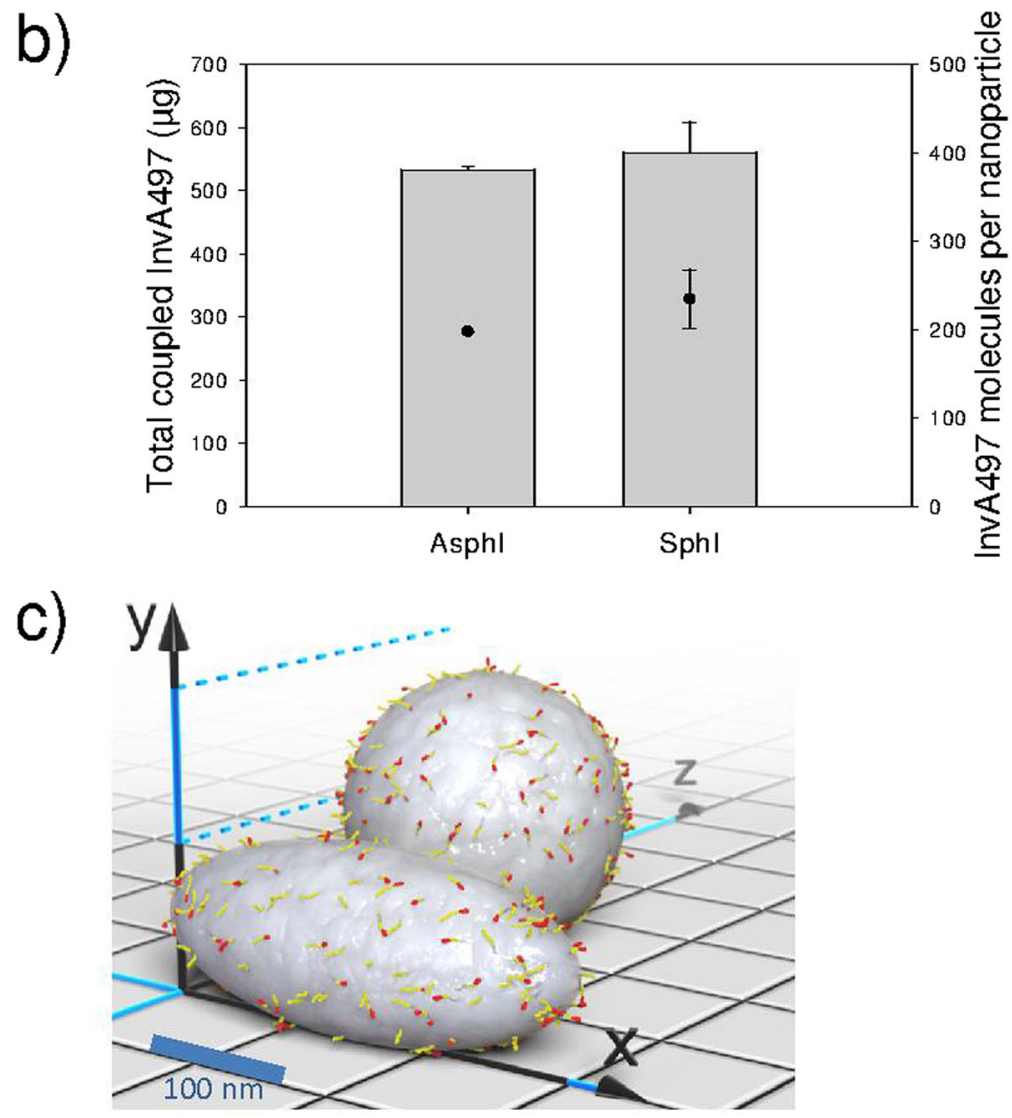

Fig. 2 Characterization of InvA497 functionalized nanoparticles. (a) Schematic of functionalization procedure of the nanoparticle surface with InvA497: carboxyl groups of spherical nanoparticles were first activated with 4-(4,6-Dimethoxy-1,3,5-triazin-2-yl)-4-methylmorpholinium chloride (DMTMM) and then InvA497 was coupled on the surface. For aspherical nanoparticles, surface coupling of InvA497 was performed after activation of spherical nanoparticle carboxylic acid groups and particle stretching; b) quantification of the total amount of coupled InvA497 (grey bars) and estimation of the number of InvA497 molecules/ nanoparticle (black circles), for aspherical (Asphl) and spherical (Sphl) nanoparticles; c) 3D model of Asphl and Sphl. The scale of the particles and the number of surface InvA497 molecules corresponds to the experimentally determined values. InvA497 molecules are shown in yellow, while their C-terminal integrinbinding regions are highlighted in red. Particle surfaces are colored grey. Where appropriate results represent the mean \pm SE $(n=3)$.

to Asph and Sph formulations (Fig. 3c and d) at the endpoint of uptake studies, as well as the localization of nanoparticles inside the cells (Fig. 3e and f).

The kinetics of particle uptake were then quantified using FACS in order to further investigate the influence of particle functionalization and shape on cellular internalization (Fig. 4a). Within the first $3 \mathrm{~h}$, uptake of InvA497-functionalized nanoparticles was found to be significantly greater than the non-functionalized carriers independent of particle shape. SphI demonstrated a slightly faster uptake than AsphI in the early stages of the study; by $t=4 \mathrm{~h}$ however, the uptake ratio had leveled out at approximately the same value of $66 \%$ and, hence, became independent of particle geometry. 
Fig. 3 Confocal microscopy images of the cellular uptake of nanoparticles. Uptake of aspherical ('Asphl', a) and spherical ('Sphl', b) nanoparticles functionalized with InvA497, as well as nonfunctionalized aspherical ('Asph', c) and spherical ('Sph', d) control nanoparticles into HEp-2 cells is shown after 5 h of incubation at $37^{\circ} \mathrm{C}$. Cross sections for $\mathrm{a}(\mathbf{e})$ and $\mathrm{b}$ (f) are also shown, demonstrating the internalization of the nanoparticles inside the HEp-2 cells. Red: HEp-2 cell membranes, green: nanoparticles, blue: HEp-2 cell nuclei. Scale bar: $20 \mu \mathrm{m}$. a)
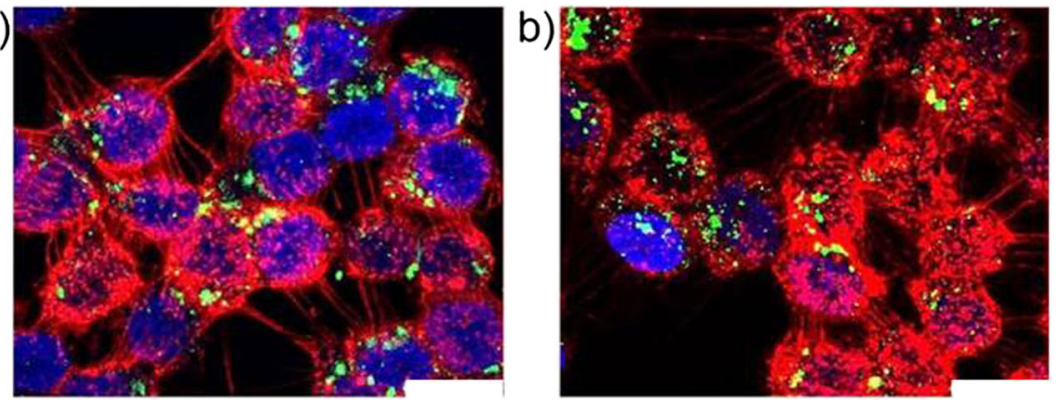

c)
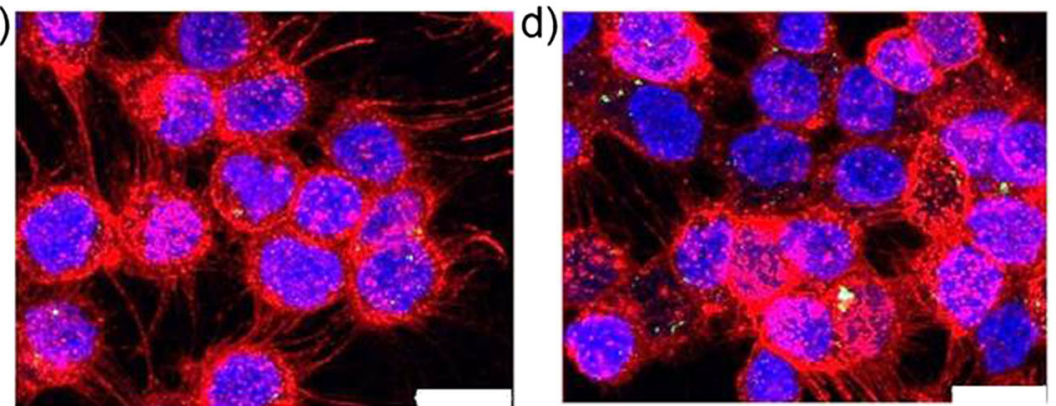

e)

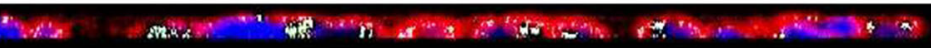

f)

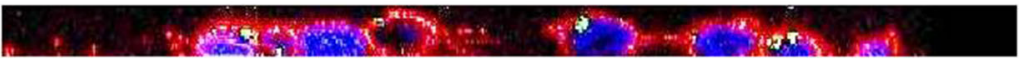

To investigate if particle uptake occurred as a result of energy dependent endocytosis or rather by membrane association, the uptake of various particle formulations into HEp-2 cells at $37^{\circ} \mathrm{C}$ and $4^{\circ} \mathrm{C}$ was compared (Fig. 4b). A significant reduction in the percentage of fluorescent cells was observed for both AsphI and SphI at $4^{\circ} \mathrm{C}$ in comparison to $37^{\circ} \mathrm{C}$.

\section{Nanoparticle Loading with AOT-Gentamicin}

Following cellular uptake investigations, nanoparticles were loaded with an anti-infective drug. In this respect, spherical AOT-gentamicin loaded (SphG) and aspherical AOTgentamicin loaded (AsphG) nanoparticles were formulated; InvA497 was also successfully coupled on the surface of drug-loaded aspherical (AsphIG) and spherical (SphIG) nanoparticles. The $\mathrm{EE} \%$ and $\mathrm{LC} \%$ of the various nanoparticle formulations is shown in Fig. 5a. The stretching procedure was seen to result in a reduction of approximately $10 \%$ with respect to the $\mathrm{EE} \%$ of AsphIG and AsphG formulations relative to their spherical counterparts, as well as a drop in LC\% of approximately $15 \%$. The amount of InvA497 present on AsphIG and SphIG surfaces was found to be comparable (Fig. 5b), and slightly higher than that of the AsphI and SphI formulations.

The size of drug-loaded nanoparticles was also found to be similar to unloaded comparators (data not shown), however a significant decrease in the measured surface charge was found for the drug loaded nanoparticles comparing with the nonloaded formulations (Fig. S3).

The in vitro release profiles of AOT-gentamicin from AsphIG, SphIG, AsphG and SphG were also measured at a pH of 7.4 (Fig. 6).

A slight difference in the kinetics of drug release as a function of nanoparticle shape was observed within the first two hours, with a greater release noted from the aspherical nanoparticles (AsphG and AsphIG) compared to the spherical ones; moreover an accompanying change in shape of these nanoparticles was observed to occur within this time period, resulting in a recovery of spherical character (Fig. S4). No difference was however noted in the extent of release from functionalized and non-functionalized carrier systems.

\section{Anti-Infective Efficacy Study}

HEp-2 cells were first infected with $S$. flexneri using different conditions (Fig. S5), in order to optimize the procedure for producing a model of intracellular infection. The invasion capacity of $S$. flexneri bacteria in different growth phases and at various MOI (multiplicity of infection - the ratio of bacterial to HEp-2 cells) was tested, in order to find parameters providing an optimal balance between invasion rate and used bacterial inoculum. HEp-2 cells were therefore infected for $2 \mathrm{~h}$ with varying amounts of bacteria, grown to either the exponential or stationary growth phase. Following infection with S. flexneri, HEp-2 cells were then treated for a further $2 \mathrm{~h}$ with 

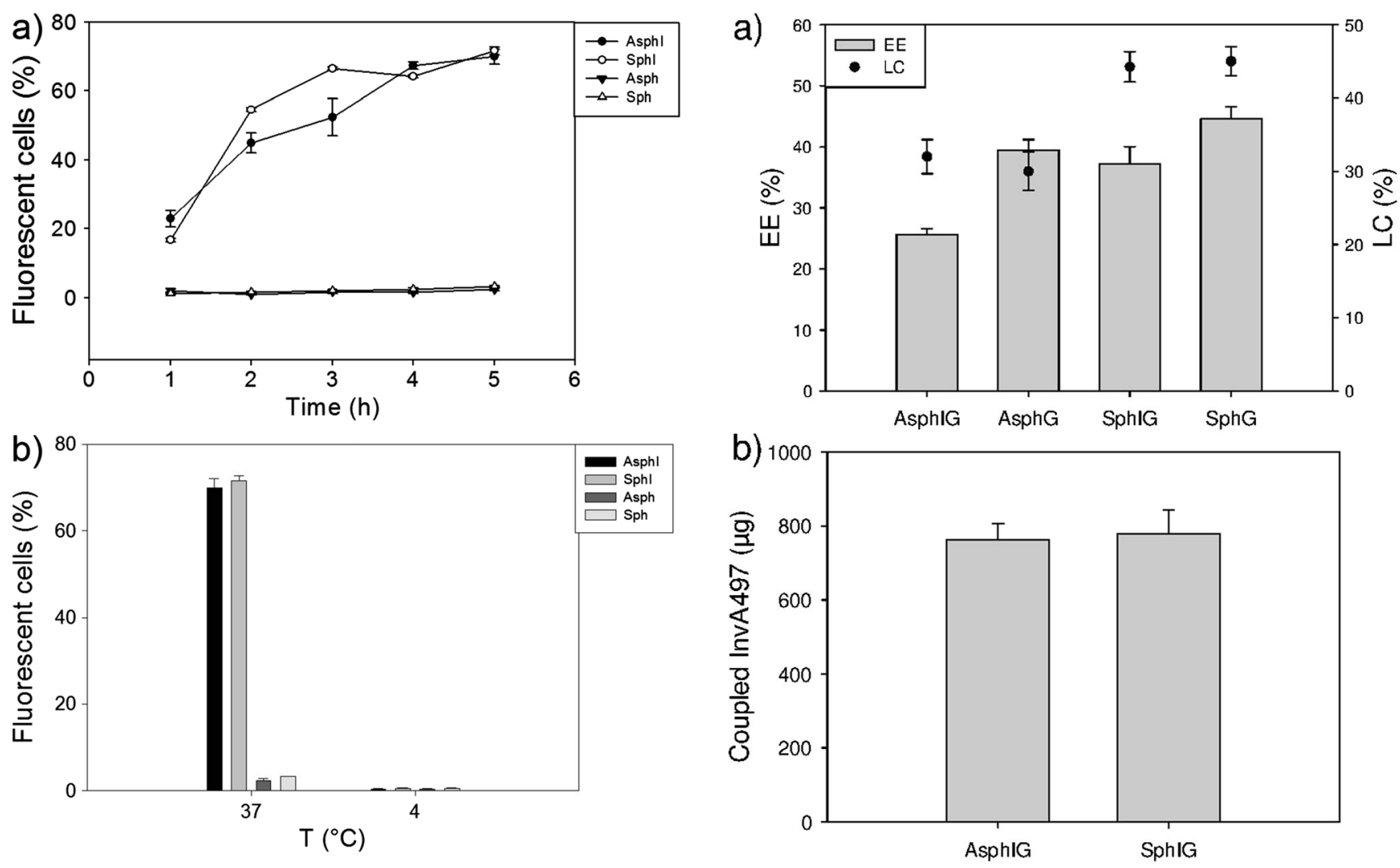

Fig. 4 Quantification of intracellular uptake of nanoparticles. a) Flow cytometry was employed to quantify nanoparticle uptake into HEp-2 cells and to analyze the uptake kinetics of aspherical InvA497-functionalized (Asphl) and spherical InvA497-functionalized (Sphl) nanoparticles, as well as non-functionalized aspherical (Asph) and spherical (Sph) particles as controls. Uptake of Asphl and Sphl was significantly greater than Asph and Sph at all investigated time points $(p<0.00 I)$. b) Uptake of Asphl, Sphl, Asph and Sph nanoparticles after $5 \mathrm{~h}$ of incubation with $\mathrm{HEp}-2$ cells at $37^{\circ} \mathrm{C}$ and $4^{\circ} \mathrm{C}$ was also investigated, in order to assess the energy dependence of particle uptake. Results represent the mean $\pm \operatorname{SE}(n=3)$.

free, unmodified (non-cell-permeable) gentamicin, in order kill any non-internalized bacteria. The application of S. flexneri grown to the exponential phase at an MOI of 25:1 was found to be the optimal condition for promoting uptake of a reproducible number of intracellular bacteria, while also avoiding overloading of HEp-2 cells (Fig. S5). After establishing an appropriate, non-toxic dose range of the various nanoparticle formulations (Fig. S6), the continued viability of HEp2 cells following both infection with S. flexneri and treatment with various nanocarriers was then tested. All nanoparticle formulations were found to be non-toxic over the employed dose range, however a reduction in cell viability was observed after administration of free AOT-gentamicin (Fig. S7). Efficacy studies were subsequently performed (Fig. 7), where HEp-2 cells intracellularly infected with $S$. flexneri were treated with freshly prepared AOT-gentamicin-loaded and InvA497functionalized nanoparticles (AsphIG and SphIG), surfacefunctionalized but drug-free aspherical and spherical nanoparticles (AsphI and SphI), AOT-gentamicin-loaded but

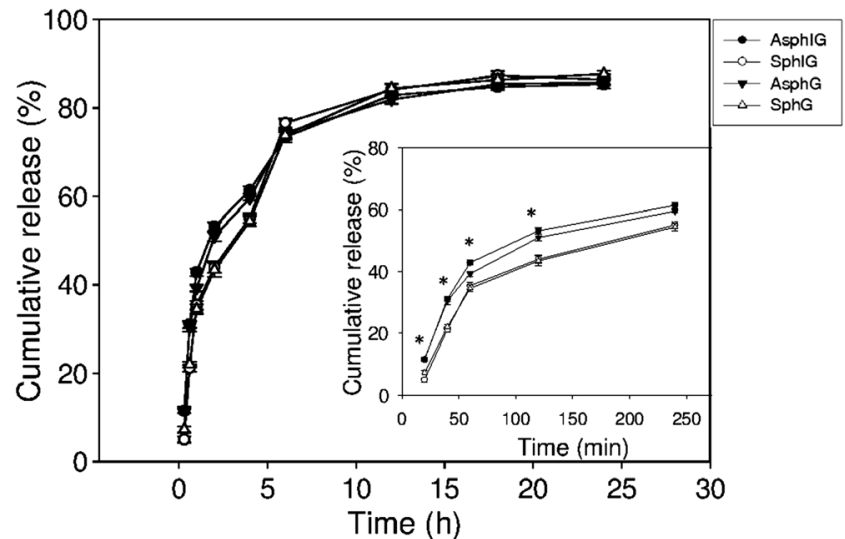

Fig. 6 In vitro release kinetics. AOT-gentamicin release was measured from aspherical (AsphlG) and spherical (SphlG) functionalized nanoparticles loaded with AOT-gentamicin, and loaded non-functionalized aspherical (AsphG) and spherical (SphG) nanoparticles over $24 \mathrm{~h}$ in PBS at $37^{\circ} \mathrm{C}$. The insert graph shows release over the first 250 min. *Indicates statistical significance with $p$ value $<0.05$ for AsphlG or Asph G versus Sphl G or SphG. Results represent the mean \pm SE of three independent experiments, each with duplicate samples. 


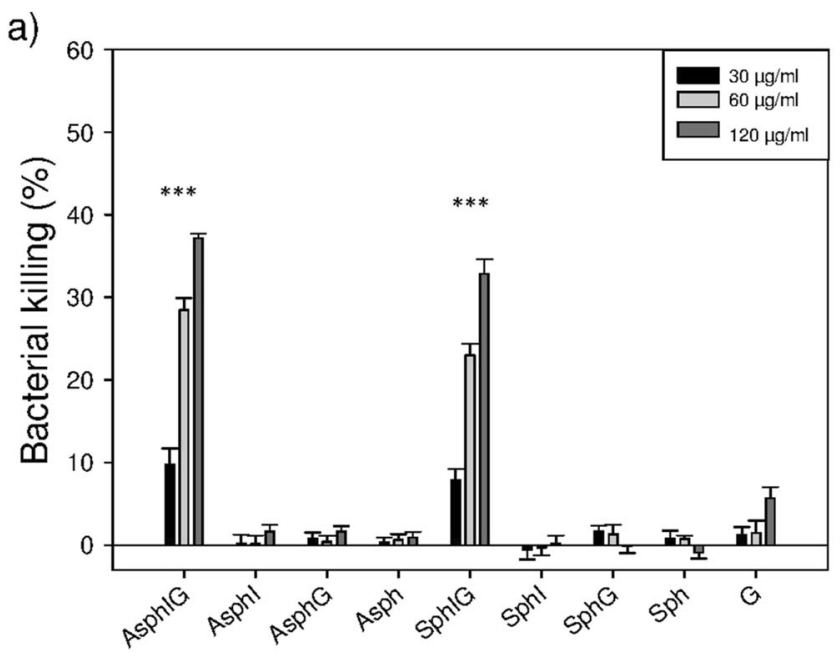

b)

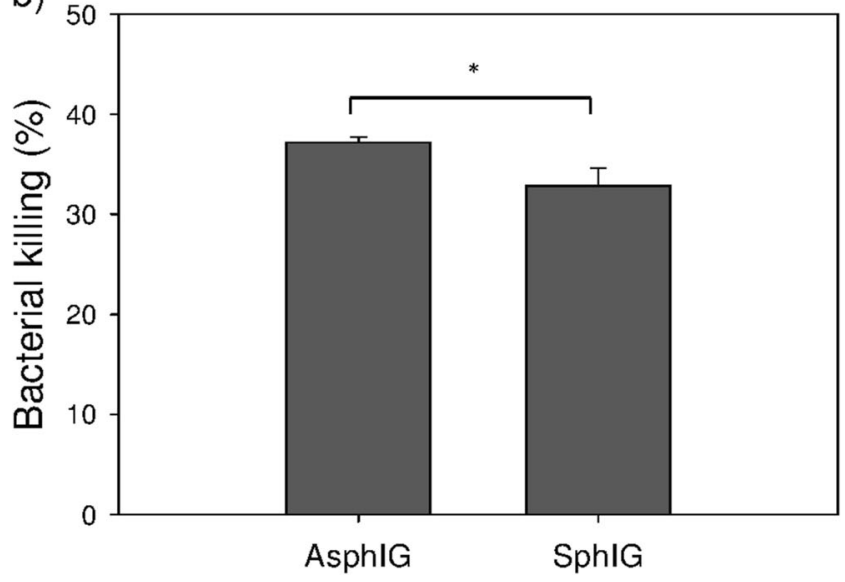

Fig. 7 Efficacy study of nanoparticles against intracellular S. flexneri. (a) Percentage of killing of intracellular S. flexneri after $2 \mathrm{~h}$ treatment with aspherical InvA497-functionalized nanoparticles loaded with AOT-gentamicin (AsphlG), aspherical InvA497-functionalized nanoparticles (Asphl), aspherical nanoparticles loaded with AOT-gentamicin (AsphG), aspherical nanoparticles (Asph), spherical InvA497-functionalized nanoparticles loaded with AOT-gentamicin (SphIG), spherical InvA497-functionalized nanoparticles (Sphl), spherical nanoparticles loaded with AOT-gentamicin (SphG), spherical nanoparticles (Sph) and AOT-gentamicin alone (G), using three different drug doses: $30 \mu \mathrm{g} / \mathrm{ml}, 60 \mu \mathrm{g} / \mathrm{ml}$ and $120 \mu \mathrm{g} / \mathrm{ml}$. *** indicates statistical significance with a p value $<0.00$ I for AsphIG versus Asphl, AsphG, Asph and G, and SphIG versus Sphl, SphG, Sph and G. (b) Direct comparison of bacterial killing of AsphIG and SphlG, at $120 \mu \mathrm{g} / \mathrm{ml}$ of AOT-gentamicin. * indicates $\mathrm{p}$ value $<0.05$. Data shows the mean \pm SE of 3 independent experiments, each employing duplicate samples.

non-functionalized nanoparticles (AsphG and $\mathrm{SphG})$, and nanoparticles without any surface functionalization or drug loading (Asph and Sph). Free AOT-gentamicin was also employed as a control (Fig. 7a).

A dose dependent reduction in the number of intracellular bacteria was seen following treatment with either AsphIG or SphIG, with the bacterial killing induced by these formulations being significantly greater than all other formulations of corresponding shape, as well as the free AOT-gentamicin. A small but significant difference in bacteria killing was also found between the AsphIG and SphIG formations at the highest tested concentration, where a greater bacterial killing was registered for AsphIG compared to SphIG (Fig. 7b).

\section{DISCUSSION}

This study was initiated to investigate whether the previously shown ability of InvA497 to mediate uptake and efficacy of anti-infective loaded, lipid-based nanocarriers could be applied to more robust, polymer-based systems. Moreover, bacteriomimetic nanoparticles with an aspherical, rod-like shape, as exhibited by a number of invasive bacteria, were also studied in order to investigate the possible role of particle shape in system efficacy.

To achieve these objectives, aspherical nanoparticles were first prepared by applying a thermomechanical stress (29) to spherical nanoparticle precursors immobilized within a polymeric film, leading to a change of the spherical shape into an elongated rod-like morphology. The use of a low ratio of FAPLGA:PLGA during spherical nanoparticle preparation ensured the production of nanoparticles with a covalently linked fluorescent label, as well as a large number of non-modified PLGA molecules available for subsequent InvA497 coupling (see below). As DLS-based size measurements rely on the assumption that detected particles have a spherical shape, the dimensions of aspherical nanoparticles were rather characterized by SEM image analysis (17). Stretching of $160 \mathrm{~nm}$ diameter Sph nanoparticles was seen to result in elongated Asph particles (Fig. 1) with an AR of approximately 2.6, a value which is comparable to the aspect ratio of Yersinia bacteria (37).

Aspherical and spherical nanoparticles were then surfacefunctionalized with InvA497, a $C$-terminal fragment of the Yersinia-derived invasion protein invasin. As observed previously $(3,5,9)$, the uptake of spherical delivery systems functionalized with InvA497 is enhanced due to the presence of this bacterial protein on particle surfaces; it was therefore of interest to see whether the same effect was noted with an aspherical delivery system. In order to bind to the $\beta_{1}$ integrin receptor on epithelial cells and mediate internalization, the $C$-terminal carboxyl group of InvA497 must remain free and project outwards from the particle surface. Therefore, amine functionalities of InvA497 were reacted with free PLGA carboxyl groups of both aspherical and spherical nanoparticles, in order to achieve protein coupling to particle surfaces without alteration of the integrin receptor-binding domain (Fig. 2a). Due to their non-reactive nature, a coupling agent, DMTMM, was first used to activate PLGA carboxyl groups, prior to addition and coupling of InvA497. AsphI and SphI formulations were found to have a similar amount of coupled InvA497 as well as percentage of surface occupancy (Fig. 2b and c), which allowed for an accurate comparison of their relative uptake. 
Before carrying out uptake studies, the cytotoxicity of AsphI, SphI, Asph and Sph in a HEp-2 epithelial cell model was preliminarily assessed at values encompassing a feasible working range. Nanoparticle concentrations resulting in a comparable InvA497 concentration to that of the previously tested InvA497-functionalized liposomes (9), and corresponding to the maximum possible concentration of dose-able InvA497 were used (Fig. S2). Nanoparticle formulations were then incubated with HEp-2 cells to determine the cellular uptake. A previous study conducted with liposomal nanoparticles functionalized with the same InvA497 invasin fragment has shown an increased cellular uptake due to the presence of InvA497 on the surface of such nanocarriers $(3,9)$; in accordance with this observation, InvA497 functionalization of both spherical and aspherical nanoparticles in the current work enhanced their uptake and internalization into epithelial cells, as confirmed by both CLSM (Fig. 3) and FACS analysis (Fig. 4). Interestingly, only slight differences in the kinetics of SphI and AsphI cellular uptake were seen, at earlier time points; no appreciable difference in the total extent of uptake was noted. This result is not entirely unexpected, given the aforementioned lack of enhancement of cellular uptake generally demonstrated by aspherical systems (18). In agreement with the previously conducted and mentioned study employing a liposomal formulation (3), InvA497-coated nanoparticles were found to be taken up via an energy-dependent mechanism (Fig. 4b).

Following the demonstration of an increase in cellular uptake of InvA497-functionalized nanoparticles, analogous nanoparticle formulations were then loaded with an antiinfective drug to enable further investigation of their properties and ultimately, their efficacy. The broad spectrum antibiotic gentamicin was employed for this purpose - however, due to the highly hydrophilic nature of gentamicin and the consequently low level of drug encapsulation within PLGA nanoparticles, a lipophilic preparation of gentamicin was used (AOT-gentamicin). Simple mixing of gentamicin and the surfactant AOT, followed by solvent evaporation, leads to formation of the lipophilized preparation, as a result of electrostatic interaction between the five amine groups of gentamicin molecules and AOT (36). After AOT-gentamicin preparation, AsphIG, SphIG, AsphG and SphG were formulated and characterized. Comparable results were found for the SphG nanoparticles and AOT-gentamicin loaded PLGA nanoparticles previously prepared by Imbuluzqueta et al. (36), with an $\mathrm{EE} \%$ and $\mathrm{LC} \%$ of 43 and $46 \%$ respectively. A slight reduction of approximately $10 \%-15 \%$ in $\mathrm{EE} \%$ and LC $\%$ was observed for aspherical as compared to spherical formulations, as a result of the stretching procedure (Fig. 5); however even with this reduction, aspherical nanoparticle formulations still demonstrated a good ability to incorporate AOT-gentamicin. The amount of InvA497 functionalization for both AsphIG and SphIG was found to be slightly higher than for the AsphI and SphI, but remained comparable between AsphIG and SphIG despite the small differences in amount of encapsulated drug.

Next, the kinetics of AOT-gentamicin release from nanocarriers was tested (Fig. 6). A marginally faster release of AOT-gentamicin was noted from the aspherical nanoparticles as compared to the spherical ones, but only during the initial stages of the study. This slightly accelerated release profile may potentially have been driven by a recovery of spherical shape, as was noted in aspherical nanoparticle samples at the conclusion of the release study (Fig. S4). It is considered unlikely that this morphological change was rather the result of PLGA degradation, as this is expected to occur over a longer time frame of several days (38). While a reversion of aspherical particles to a spherical morphology has been previously reported (39), it has not, to the best of the authors' knowledge, been observed to date to correlate with an accelerated release behavior; this could of course also influence the cellular uptake of such carriers, and as such constitutes a point of considerable further research interest.

In a final stage, the efficacy of functionalized and drugloaded spherical and aspherical nanoparticles against intracellular $S$. flexneri was tested, in order to compare and ultimately evaluate the full potential of the bacteriomimetic delivery systems against a pathogen of clinical importance (40). Optimization of infection conditions and employed formulation dose was carried out prior to the conduction of efficacy studies themselves (Figs. S5 and S6). Interestingly, a negative effect on HEp-2 cell viability was seen in these optimization studies when infected cells were treated with $120 \mu \mathrm{g} / \mathrm{ml}$ of free AOT-gentamicin compared with all AOT-gentamicin loaded nanoparticles, indicating a benefit of incorporating AOTgentamicin into nanoparticles regardless of their shape and surface functionalization (Fig. S7). In the efficacy study itself, AsphIG and SphIG induced a higher bacterial killing than all other tested formulations, with a dose dependent killing effect being observed (Fig. 7a). Notably, a small but significant improvement of bacterial killing was seen with AsphIG as compared to SphIG at the highest employed dose (Fig. 7b). This difference indicates a promising application of shape-modified InvA497-functionalized carrier systems for intracellular infection treatment; however the modest magnitude of this difference highlights the considerable remaining scope for carrier system development and probing of shape effects - namely, optimization of aspect ratio and maintenance of asphericity in order to further enhance intracellular bacterial killing.

Taken together, these results demonstrate that the presence of InvA497 on the surface of polymer-based nanocarriers in combination with encapsulation of AOTgentamicin is able to increase the ability of the delivery system to effectively treat infected epithelial cells. The obtained data on bacterial killing indicate that carrier shape may potentially have an influence on the efficacy 
of bacteriomimetic delivery systems; regardless of shape however, functionalization of polymeric systems with InvA497 presents a delivery option where the advantage of being actively transported across cell membranes in order to access intracellular bacteria is combined with an ability to release drug cargo within the intracellular environment.

\section{CONCLUSION}

In summary, we have shown that InvA497 can successfully mediate the cellular internalization and efficacy of polymeric carrier systems against intracellular bacterial infections. So-called bacteriomimetic nanocarriers, surface functionalized with InvA497 and possessing either a spherical or aspherical shape, showed a better cellular uptake compared to nanocarriers without InvA497. Anti-infective loaded bacteriomimetic systems further demonstrated a markedly improved killing of intracellular $S$. flexneri in comparison to non-functionalized systems. The small but significant improvement in killing of intracellular S. flexneri resulting from treatment with a high dose of aspherical bacteriomimetic systems as compared to spherical is of great interest for future work. Subsequent work will therefore focus on the further optimization of robust, polymer-based bacteriomimetic systems. This will include greater exploration of the ability to enhance the impact of shape factors, as well as application of these systems for the control of intracellular pathogens and their respective diseases typical of specific routes of administration, such as the gastro-intestinal and pulmonary tract.

\section{ACKNOWLEDGMENTS AND DISCLOSURES}

The authors thank Dr. Cristiane de Souza Carvalho Wodarz for her advice and assistance with the bacterial efficacy studies. Dr. Sabrina Muehlen and Carina Schmühl are gratefully acknowledged for support and training related to bacterial culture and invasion protocols. Tanja Krause is also acknowledged for the InvA497 purification.

Open Access This article is distributed under the terms of the Creative Commons Attribution 4.0 International License (http://creativecommons.org/licenses/by/4.0/), which permits unrestricted use, distribution, and reproduction in any medium, provided you give appropriate credit to the original author(s) and the source, provide a link to the Creative Commons license, and indicate if changes were made.

Publisher's note Springer Nature remains neutral with regard to jurisdictional claims in published maps and institutional affiliations.

\section{REFERENCES}

1. Sansonetti P. Bacterial pathogens, from adherence to invasion: comparative strategies. Med Microbiol Immunol. 1993;182(5): 223-32.

2. Phalipon A, Sansonetti PJ. Shigella's ways of manipulating the host intestinal innate and adaptive immune system: a tool box for survival? Immunol Cell Biol. 2007;85(2):119-29.

3. Labouta HI, Menina S, Kochut A, Gordon S, Geyer R, Dersch P, et al. Bacteriomimetic invasin-functionalized nanocarriers for intracellular delivery. J Controlled Release. 2015;220(Pt A):414-24.

4. Salman HH, Gómez S, Gamazo C, Costa Martins R, Zabaleta V, Irache JM. Micro-organism-like nanoparticles for oral antigen delivery. J Drug Deliv Sci Technol. 2008;18(1):31-9.

5. Dawson GF, Halbert GW. The in vitro cell association of invasin coated polylactide-co-glycolide nanoparticles. Pharm Res. 2000;17(11):1420-5.

6. Hussain N, Florence AT. Utilizing bacterial mechanisms of epithelial cell entry: Invasin-induced oral uptake of latex nanoparticles. Pharm Res. 1998;15(1):153-6.

7. Wiedemann A, Linder S, Grassl G, Albert M, Autenrieth I, Aepfelbacher M. Yersinia enterocolitica invasin triggers phagocytosis via betal integrins, CDC42Hs and WASp in macrophages. Cellular Microbiol. 2001;3(10):693-702.

8. Dersch P, Isberg RR. A region of the Yersinia pseudotuberculosis invasin protein enhances integrin-mediated uptake into mammalian cells and promotes self-association. EMBO J. 1999;18(5):1199-213.

9. Menina S, Labouta HI, Geyer R, Krause T, Gordon S, Dersch P, et al. Invasin-functionalized liposome nanocarriers improve the intracellular delivery of anti-infective drugs. RSC Adv. 2016;6(47):41622-9.

10. Justice SS, Hunstad DA, Cegelski L, Hultgren SJ. Morphological plasticity as a bacterial survival strategy. Nat Rev Microbiol. 2008;6(2):162-8.

11. Young KD. Bacterial morphology: why have different shapes? Curr Opin Microbiol. 2007;10(6):596-600.

12. Truong NP, Whittaker MR, Mak CW, Davis TP. The importance of nanoparticle shape in cancer drug delivery. Expert Opin Drug Deliv. 2015;12(1):1-14.

13. Simone EA, Dziubla TD, Muzykantov VR. Polymeric carriers: role of geometry in drug delivery. Expert Opin Drug Deliv. 2008;5(12): 1283-300.

14. Decuzzi P, Godin B, Tanaka T, Lee SY, Chiappini C, Liu X, et al. Size and shape effects in the biodistribution of intravascularly injected particles. J Control Release. 2010;141(3):320-7.

15. Cooley M, Sarode A, Hoore M, Fedosov DA, Mitragotri S, Sen GA. Influence of particle size and shape on their margination and wall-adhesion: implications in drug delivery vehicle design across nano-to-micro scale. Nanoscale. 2018;10(32):15350-64.

16. Ghampion JA, Mitragotri S. Shape induced inhibition of phagocytosis of polymer particles. Pharm Res. 2009;26(1):244-9.

17. Herd H, Daum N, Jones AT, Huwer H, Ghandehari H, Lehr C-M. Nanoparticle geometry and surface orientation influence mode of cellular uptake. ACS Nano. 2013;7(3):1961-73.

18. Mathaes R, Winter G, Besheer A, Engert J. Influence of particle geometry and PEGylation on phagocytosis of particulate carriers. Int J Pharm. 2014;465(1-2):159-64.

19. Mathaes R, Winter G, Siahaan TJ, Besheer A, Engert J. Influence of particle size, an elongated particle geometry, and adjuvants on dendritic cell activation. Eur J Pharm Biopharm. 2015;94:542-9.

20. Li D, Zhuang J, He H, Jiang S, Banerjee A, Lu Y, et al. Influence of particle geometry on gastrointestinal transit and absorption following Oral administration. ACS Appl Mater Interfaces. 2017;9(49): 42492-502.

21. Möhwald M, Pinnapireddy SR, Wonnenberg B, Pourasghar M, Jurisic M, Jung A, et al. Aspherical, nanostructured microparticles 
for targeted gene delivery to alveolar macrophages. Adv Health Mater. 2017;6(20):1700478-n/a.

22. Champion JA, Mitragotri S. Role of target geometry in phagocytosis. Proc Natl Acad Sci U S A. 2006;103(13):4930-4.

23. Banerjee A, Qi J, Gogoi R, Wong J, Mitragotri S. Role of nanoparticle size, shape and surface chemistry in oral drug delivery. J Control Release. 2016;238:176-85.

24. Barua S, YooJ-W, Kolhar P, Wakankar A, Gokarn YR, Mitragotri S. Particle shape enhances specificity of antibody-displaying nanoparticles. Proc Natl Acad Sci U S A. 2013;110(9):3270-5.

25. Kolhar P, Anselmo AC, Gupta V, Pant K, Prabhakarpandian B, Ruoslahti E, et al. Using shape effects to target antibody-coated nanoparticles to lung and brain endothelium. Proc Natl Acad Sci U S A. 2013;110(26):10753-8.

26. Hinde E, Thammasiraphop K, Duong HTT, Yeow J, Karagoz B, Boyer $\mathrm{C}$, et al. Pair correlation microscopy reveals the role of nanoparticle shape in intracellular transport and site of drug release. Nat Nanotechnol. 2017;12(1):81-9.

27. Haque S, Boyd BJ, McIntosh MP, Pouton GW, Kaminskas LM, Whittaker M. Suggested procedures for the reproducible synthesis of poly(d,l-lactide-co-glycolide) nanoparticles using the emulsification solvent diffusion platform. Curr Nanosci. 2018;14(5):448-53.

28. Weiss B, Schaefer UF, Zapp J, Lamprecht A, Stallmach A, Lehr GM. Nanoparticles made of fluorescence-labelled poly(L-lactideco-glycolide): preparation, stability, and biocompatibility. J Nanosci Nanotechnol. 2006;6(9-10):3048-56.

29. Champion JA, Katare YK, Mitragotri S. Making polymeric microand nanoparticles of complex shapes. Proc Natl Acad Sci U S A. 2007;104(29):11901-4.

30. Ho CG, Keller A, Odell JA, Ottewill RH. Preparation of monodisperse ellipsoidal polystyrene particles. Colloid Polym Sci. 1993;271(5):469-79.
31. Dersch P, Isberg RR. An immunoglobulin superfamily-like domain unique to the Yersinia pseudotuberculosis invasin protein is required for stimulation of bacterial uptake via integrin receptors. Infect Immun. 2000;68(5):2930-8.

32. Smith PK, Krohn RI, Hermanson GT, Mallia AK, Gartner FH, Provenzano MD, et al. Measurement of protein using bicinchoninic acid. Anal Biochem. 1985;150(1):76-85.

33. Hamburger ZA, Brown MS, Isberg RR, Bjorkman PJ. Crystal structure of Invasin: a bacterial integrin-binding protein. Science. 1999;286(5438):291-5.

34. Krieger E, Koraimann G, Vriend G. Increasing the precision of comparative models with YASARA NOVA-a self-parameterizing force field. Proteins. 2002;47(3):393-402.

35. Elizondo E, Sala S, Imbuluzqueta E, Gonzalez D, Blanco-Prieto MJ, Gamazo C, et al. High loading of gentamicin in bioadhesive PVM/MA nanostructured microparticles using compressed carbon-dioxide. Pharm Res. 2011;28(2):309-21.

36. Imbuluzqueta E, Elizondo E, Gamazo C, Moreno-Calvo E, Veciana J, Ventosa N, et al. Novel bioactive hydrophobic gentamicin carriers for the treatment of intracellular bacterial infections. Acta Biomater. 201 1;7(4):1599-608.

37. Hoekstra A, Maltsev V, Videen G. Optics of biological particles: Springer Netherlands; 2007.

38. Park TG. Degradation of poly(lactic-co-glycolic acid) microspheres: effect of copolymer composition. Biomaterials. 1995;16(15): 1123-30.

39. Yoo JW, Mitragotri S. Polymer particles that switch shape in response to a stimulus. Proc Natl Acad Sci U S A. 2010;107(25): $11205-10$

40. Kotloff KL, Riddle MS, Platts-Mills JA, Pavlinac P, Zaidi AKM. Shigellosis. Lancet. 2018;391(10122):801-12. 